\title{
Article \\ Higher dimensional Bianchi Type-III String Cosmological Models in Lyra Geometry
}

\author{
Kangujam Priyokumar Singh ${ }^{1,(0)}$, Jiten Baro ${ }^{2}$, S. Romaleima Devi ${ }^{3}$ and Asem Jotin Meitei ${ }^{4 *(1)}$ \\ 1 Department of Mathematics, Manipur University, Imphal-795003, Manipur, India; \\ pk_mathematics@yahoo.co.in \\ 2 Department of Mathematical Sciences, Bodoland University \\ Kokrajhar-783370, BTC, Assam, India; barojiten5@gmail.com \\ 3 Department of Mathematics, Manipur College \\ Imphal, Manipur-795008, India; s.romaleima@gmail.com \\ 4 Department of Mathematics, Pravabati College, Mayang Imphal-795132, Manipur, India; \\ jotinasem@gmail.com \\ * Correspondence: jotinasem@gmail.com; Tel.: (+919774571821)
}

\begin{abstract}
Here we studied Bianchi type-III string cosmological models generated by means of a cloud of strings with particles connected to them in the framework of Lyra geometry considering five-dimensional space-time. To obtain the exact solutions of field equations we consider that the shear scalar and the scalar expansion are proportional, $\sigma \infty \theta$ which leads to $D=c^{n}$ and secondly we adopt the assumption considering the Reddy String Condition, $\rho+\lambda=0$. From the two different cases obtained here, first case leads to the Bianchi type-III string cosmological model in Lyra geometry in five-dimensional space-time and the second case leads to the five-dimensional vacuum universe in general relativity. The Geometrical and physical properties of the model universe are studied comparing with the present day's observational findings. The model universe obtained here starts with the big bang and as time progresses both particle density $\rho_{p}$ and energy density $\rho$ decreases with expansion of our Universe.
\end{abstract}

Keywords: Five dimension, Cloud String, Bianchi Type-III Space-Time , Lyra Geometry, Expansion Scalar and Big-bang

\section{Introduction}

It is still an interesting area of research to discover its unknown phenomenons that have yet to observed to study the ultimate fate of the Universe. So cosmologists have taken considerable interest to understand the future evolution of the Universe and comprehension of the past and present state of the Universe and to understand about the universe in modern era. Recently, there has been considerably interest to study the string cosmology because of the important role of cosmic strings to study about the origin and evolution of the universe. But till these days we can not make a very last assertion about its origin and evolution with strong evidence. So more and more investigations are very important to discover the unknown phenomenons of the whole universe. The string theory was developed to describe the events of the early stage of origin and evolution of the Universe. Generally the study of string was started by Stachel[1] and Letelier[2]. Because of the key function of strings in describing the evolution of the early stage of our universe, in recent times, many prominent authors are interested in cosmic strings in general relativity (Kibble[3], Kibble[4]) and give rise to density perturbations leading to the formation of large Scale Structure (galaxies) of the universe (Zel'dovich [5], Zel'dovich [6]). The strings are nothing but crucial topological defects because of the phase transition which occurs at the temperature lower than some critical point at the beginning of our universe according to grand unified theories (Vilenkin [7], Vilenkin [8]). Also, a large network of strings present in early 
universe does not oppose present day observational findings of the Universe and results in an anisotropy in the space-time though the strings are not observable in the present day's universe. Those strings have stress energy and they may be coupled with the gravitational field. The Strings don't cause any harm to the cosmological models, rather it can lead to very interesting astrophysical phenomena.

Among the modified theories of gravity, Lyra geometry is also one of the important theory in which the displacement field can be considered as a component of the total energy that can play the role of dark energy. Lyra[9] suggested a modification to Riemannian geometry by using a gauge function $\phi_{\mu}$ into the structureless geometry which removed the non integrability condition to H. Weyl's [10] geometry and this modified Riemannian geometry by Lyra is known as Lyra's Geometry. Halford [11] Constructed a cosmological theory in the framwork of Lyra Manifold and Mentioned that the displacement vector in Lyra manifold plays the position of cosmological constant $\Lambda$ in general relativity. Some of the prominent researchers in the area of Lyra geometry are Rahaman and Bera [10], Rahaman et al. [11], Singh et al.[12], Mohanty et al.[13], [14], G. Mohanty and K. L. Mahanta[15], Singh and Mollah[16], who constructed various cosmological models universe in the context of Lyra geometry.

Many researchers have studied different string cosmological models in different dimensions. It is believed that the universe had some higher dimensional era at its early stage of evolution which motivates us to investigate the cosmological model universe in five dimensional space-time. Nowadays it is interesting to study string cosmology in five dimensional space time in the framework of general relativity as well as in Lyra geometry. Rahaman et al.[17] obtained realistic solutions of the field equations in Lyra geometry for a five dimensional space time when the gravitational sources are massive strings. Mohanty and Samanta[18] constructed five dimensional string cosmological models in Lyra manifold with massive scalar field and obtained that the models avoid initial singularity in presence of massive scalar field.Yadav et al.[19] have investigated Bianchi type-III anisotropic universes with cloud of strings within the framework of Lyra geometry. Samanta et al.[20] constructed some Bianchi type-III cosmological models in the framework of general relativity considering five dimensional space time with massive string as a source of gravitational field.Adhav et al.[21], Kandalker et al.[22] constructed string cosmological models by considering the Reddy string condition $(\rho+\lambda=0)$ for solving the field equations. Sahoo et al.[23] investigated Bianchi type-III String cosmological model with bulk viscous fluid in Lyra manifold. Reddy[24],[25], Khadekar[26], Rao[27], Yadav[28], Goswami[29], Singh[30] are the some authors who studied on different cosmological models with various modified theories of relativity in different context with different Bianchi type space-times. Not only the above mentioned authors, recently Tripathi[31], Panov[32], Pradhan[33], Tiwari et al.[34], Ram et al.[35], Mollah et al.[36] constructed string cosmological models in various contexts and in various space-times.

Motivated by the above discussions and investigations in Einstein's theory and its alternative theories of gravity, here we have studied the five dimensional string cosmological models in Bianchi type-III space-time considering Lyra geometry. The model, presented here is some what different from the earlier findings in many area and are physically realistic as the present day observational data. The paper is planed as follows:In Section 1, a brief introduction of strings and its importance and review of Lyra Geometry are discussed. In Section 2, the five dimensional Bianchi type-III metric is presented and the field equations in the framework of Lyra geometry are derived; Section 3 , deals with the determinate solutions of the field equations determined by using some physical plausible conditions and Physical and geometrical properties of our model universe with the help of graph are presented in the Section 4; In Section 5 conclusions 
are presented.

\section{The Metric and Field Equations}

We consider the five-dimensional Bianchi type-III metric as

$$
d s^{2}=-d t^{2}+a^{2} d x^{2}+b^{2} e^{-2 x} d y^{2}+c^{2} d z^{2}+D^{2} d m^{2}
$$

Where $a, b, c$ and $D$ are the functions of ' $t$ ' only. Here the extra coordinate is taken to be space-like.

For the above metric let

$$
x^{1}=x, x^{2}=y, x^{3}=z, x^{4}=m \text { and } x^{5}=t
$$

The field equations in the normal gauge for Lyra manifold as obtained by Sen[37] is

$$
R_{i j}-\frac{1}{2} g_{i j} R+\frac{3}{2} \phi_{i} \phi_{j}-\frac{3}{4} g_{i j} \phi_{k} \phi^{k}=-T_{i j}
$$

Where in geometrized unit $8 \pi G=1, C=1$ and $\phi_{i}$ is the displacement field vector defined by

$$
\phi_{i}=(0,0,0,0, \beta(t))
$$

The energy-momentum tensor for a cosmic string is

$$
T_{i j}=\rho u_{i} u_{j}-\lambda x_{i} x_{j}
$$

Where, $\rho=\rho_{p}+\lambda$ is the energy density for a cloud of string with particle attached to them, $\rho_{p}$ is the rest energy density of particles and $\lambda$ is the string tension density of cloud of string. The fifth coordinate is taken to be space like and the coordinate are co-moving. where $u^{i}$ is the five velocity vector of particles given by

$$
u^{i}=(0,0,0,0,1) \text { and } x^{i}=\left(0,0, c^{-1}, 0,0\right)
$$

Such that,

$$
u_{i} u^{j}=-1=-x_{i} x^{j} \text { and } u_{i} x^{i}=0
$$

The field equation (3) with the equations (4)-(7) for the metric (1) takes the form

$$
\begin{gathered}
\frac{\ddot{b}}{b}+\frac{\ddot{c}}{c}+\frac{\ddot{D}}{D}+\frac{\dot{b} \dot{c}}{b c}+\frac{\dot{b} \dot{D}}{b D}+\frac{\dot{c} \dot{D}}{c D}+\frac{3}{4} \beta^{2}=0 \\
\frac{\ddot{a}}{a}+\frac{\ddot{c}}{c}+\frac{\ddot{D}}{D}+\frac{\dot{a} \dot{c}}{a c}+\frac{\dot{a} \dot{D}}{a D}+\frac{\dot{c} \dot{D}}{c D}+\frac{3}{4} \beta^{2}=0 \\
\frac{\ddot{a}}{a}+\frac{\ddot{b}}{b}+\frac{\ddot{D}}{D}+\frac{\dot{a} \dot{b}}{a b}+\frac{\dot{a} \dot{D}}{a D}+\frac{\dot{b} \dot{D}}{b D}+\frac{3}{4} \beta^{2}-\frac{1}{a^{2}}=\lambda \\
\frac{\ddot{a}}{a}+\frac{\ddot{b}}{b}+\frac{\ddot{c}}{c}+\frac{\dot{a} \dot{b}}{a b}+\frac{\dot{a} \dot{c}}{a c}+\frac{\dot{b} \dot{c}}{b c}+\frac{3}{4} \beta^{2}-\frac{1}{a^{2}}=0 \\
\frac{\dot{a} \dot{b}}{a b}+\frac{\dot{a} \dot{c}}{a c}+\frac{\dot{a} \dot{D}}{a D}+\frac{\dot{b} \dot{c}}{b c}+\frac{\dot{b} \dot{D}}{b D}+\frac{\dot{c} \dot{D}}{c D}-\frac{3}{4} \beta^{2}-\frac{1}{a^{2}}=\rho \\
\frac{\dot{a}}{a}-\frac{\dot{b}}{b}=0
\end{gathered}
$$

Where the overhead dots denotes differentiation with respect to time ' $t$ '. 


\section{Solution of the Field Equations}

From equation (13) we have

$$
a=l b
$$

where $l$ is the constant of integration. Without loss of generality we take $l=1$.

$$
a=b
$$

Thus using relation (15) the field equations (8) to (12) reduces to

$$
\begin{gathered}
\frac{\ddot{b}}{b}+\frac{\ddot{c}}{c}+\frac{\ddot{d}}{d}+\frac{\dot{b} \dot{c}}{b c}+\frac{\dot{b} \dot{D}}{b D}+\frac{\dot{c} \dot{D}}{c D}+\frac{3}{4} \beta^{2}=0 \\
2 \frac{\ddot{b}}{b}+\frac{\ddot{D}}{D}+\frac{\dot{b}^{2}}{b^{2}}+2 \frac{\dot{b} \dot{D}}{b D}+\frac{3}{4} \beta^{2}-\frac{1}{b^{2}}=\lambda \\
2 \frac{\ddot{b}}{b}+\frac{\ddot{c}}{c}+\frac{\dot{b}^{2}}{b^{2}}+2 \frac{\dot{b} \dot{c}}{b c}+\frac{3}{4} \beta^{2}-\frac{1}{b^{2}}=0 \\
\frac{\dot{b}^{2}}{b^{2}}+2 \frac{\dot{b} \dot{c}}{b c}+2 \frac{\dot{b} \dot{D}}{b D}+\frac{\dot{c} \dot{D}}{c D}-\frac{3}{4} \beta^{2}-\frac{1}{b^{2}}=\rho
\end{gathered}
$$

We have 4 independent equations (16)-(19) with Six unknowns variables b, $c, D, \beta, \lambda, \rho$. For deterministic solution we considered the following physical plausible conditions: (i) Here, we assume that the shear scalar and the scalar expansion are proportional to each other, $\sigma \infty \theta$ which leads to

$$
D=c^{n}
$$

where $n \neq 0$ is a constant.

This condition is assumed based on Thorne[38] and the observations of the velocity red-shift relation for extragalactic sources suggested that the Hubble expansion of the universe is isotropic to within 30 percent today[Kantowski and Sachs[39], Kristian and Sachs[40]]. If $\sigma$ is shear and $\mathrm{H}$ is a Hubble constant, the redshift studies limit $\frac{\sigma}{H} \leq 0.30$. If $\theta$ is expansion in the model then, for spatially homogeneous metric, the normal congruence to the homogeneous hypersurface that satisfies the condition $\frac{\sigma}{\theta}$ is constant[Collins et al.[41]].

(ii)we also adopt the assumption considering the Reddy String Condition[Reddy[42], [43], Reddy and Rao[44], [45]].

$$
\rho+\lambda=0
$$

which is the most suitable condition to explore the physically meaningful solutions of the above field equations.

Now subtracting (16) from (18) yields

$$
\frac{\ddot{b}}{b}-\frac{\ddot{D}}{D}+\frac{\dot{b}^{2}}{b^{2}}+\frac{\dot{b} \dot{c}}{b c}-\frac{\dot{b} \dot{D}}{b D}-\frac{\dot{c} \dot{D}}{c D}-\frac{1}{b^{2}}=0
$$

Adding (17) and (19) and using (21) yields,

$$
2 \frac{\ddot{b}}{b}+\frac{\ddot{D}}{D}+2 \frac{\dot{b}^{2}}{b^{2}}+2 \frac{\dot{b} \dot{c}}{b c}+4 \frac{\dot{b} \dot{D}}{b D}+\frac{\dot{c} \dot{D}}{c D}-2 \frac{1}{b^{2}}=0
$$

Equations (22) and (23) yields

$$
\frac{\ddot{D}}{D}+2 \frac{\dot{b} \dot{D}}{b D}+\frac{\dot{c} \dot{D}}{c D}=0
$$


By using (20) in (24), we get

$$
\frac{\dot{c}}{c}\left[\frac{\ddot{c}}{\dot{c}}+n \frac{\dot{c}}{c}+2 \frac{\dot{b}}{b}\right]=0
$$

Which yields the following three cases:

CaseI: $\frac{\ddot{c}}{\dot{c}}+n \frac{\dot{c}}{c}+2 \frac{\dot{b}}{b}=0$

CaseII: $\frac{\dot{c}}{c}=0$

CaseIII: $\frac{\dot{c}}{c}=0$ and $\frac{\ddot{c}}{\dot{c}}+n \frac{\dot{c}}{c}+2 \frac{\dot{b}}{b}=0$

In the following subsections we intend to determined the cosmic string cosmological models for the above mentioned three cases separately.

3.1 CaseI: $\frac{\ddot{c}}{\dot{c}}+n \frac{\dot{c}}{c}+2 \frac{\dot{b}}{b}=0$.

Solving we get,

$$
c(t)=\left[(n+1)\left(\int \frac{k}{b^{2}(t)} d t+K_{1}\right)\right], n \neq 1
$$

From this it is clear that $\mathrm{c}(\mathrm{t})$ can be find for any given function $\mathrm{b}(\mathrm{t})$. So the solution are not unique. However for further studies here we consider[Mohanty and Mahanta[15]],

$$
\frac{\ddot{c}}{\dot{c}}+n \frac{\dot{c}}{c}=-2 \frac{\dot{b}}{b}=k(\text { Constant })
$$

Now solving (27) and using (15) and (20) we get,

$$
\begin{gathered}
a=b=k_{1} e^{-\frac{k t}{2}} \\
c=\left[(n+1)\left(\frac{k_{2}}{k} e^{k t}+k_{3}\right)\right]^{\frac{1}{n+1}}, n \neq-1 \\
D=\left[(n+1)\left(\frac{k_{2}}{k} e^{k t}+k_{3}\right)\right]^{\frac{n}{n+1}}, n \neq-1
\end{gathered}
$$

where $k_{1}, k_{2}$ and $k_{3}(\neq 0)$ are constants of integration.

The geometry of the model is described by the metric

$$
\begin{aligned}
d s^{2} & =-d t^{2}+k_{1}^{2} e^{-k t}\left(d x^{2}+e^{-2 x} d y^{2}\right)+\left[(n+1)\left(\frac{k_{2}}{k} e^{k t}+k_{3}\right)\right]^{\frac{2}{n+1}} d z^{2} \\
& +\left[(n+1)\left(\frac{k_{2}}{k} e^{k t}+k_{3}\right)\right]^{\frac{2 n}{n+1}} d m^{2}
\end{aligned}
$$

Using (28), (29) and (30) in the equation (16) yields

$$
\frac{3}{4} \beta^{2}=\frac{n k_{2}{ }^{2} e^{2 k t}}{(n+1)^{2}\left(\frac{k_{2}}{k} e^{k t}+k_{3}\right)^{2}}-\frac{k k_{2} e^{k t}}{2\left(\frac{k_{2}}{k} e^{k t}+k_{3}\right)}-\frac{k^{2}}{4}
$$

The Energy density of the string is obtained by using (28), (29) (30), and (32) in the equation (19)

$$
\rho=\frac{k^{2}}{2}-\frac{k k_{2} e^{k t}}{2\left(\frac{k_{2}}{k} e^{k t}+k_{3}\right)}-\frac{e^{k t}}{k_{1}^{2}}
$$

From (21), the Tension density is given by

$$
\lambda=\frac{k k_{2} e^{k t}}{2\left(\frac{k_{2}}{k} e^{k t}+k_{3}\right)}+\frac{e^{k t}}{k_{1}^{2}}-\frac{k^{2}}{2}
$$


The rest Energy density of particles attached to the string is given by

$$
\rho_{p}=k^{2}-\frac{k k_{2} e^{k t}}{\left(\frac{k_{2}}{k} e^{k t}+k_{3}\right)}-\frac{2 e^{k t}}{k_{1}^{2}}
$$

The spatial volume is given by

$$
V=\frac{(n+1) k_{1}^{2}\left(\frac{k_{2}}{k} e^{k t}+k_{3}\right)}{e^{2 k t}}
$$

The expansion scalar is given by

$$
\theta=\frac{k_{2} e^{k t}}{\left(\frac{k_{2}}{k} e^{k t}+k_{3}\right)}-k
$$

The Hubble parameter is

$$
H=\frac{k_{2} e^{k t}}{4\left(\frac{k_{2}}{k} e^{k t}+k_{3}\right)}-\frac{k}{4}
$$

The deceleration parameter is

$$
q=-\left[\frac{4 k_{2} e^{k t}}{k k_{3}}+1\right]
$$

The shear scalar of the model is

$$
\sigma^{2}=\frac{1}{8}\left[k^{2}+\frac{k_{2}^{2} e^{2 k t}\left(3 n^{2}-2 n+3\right)}{(n+1)^{2}\left(\frac{k_{2}}{k} e^{k t}+k_{3}\right)^{2}}+\frac{k k_{2} e^{k t}}{\left(\frac{k_{2}}{k} e^{k t}+k_{3}\right)}\right]
$$

3.2.CaseII: $\frac{\dot{c}}{c}=0$

Which on integration yields

$$
c=k_{4}
$$

where $k_{4}$ is constant of integration.

Equation (20) and (41) together yields

$$
D=k_{4}^{n}
$$

Now field equations(16)-(19) with the help of (41) and (42) reduces to

$$
\begin{gathered}
\frac{\ddot{b}}{b}+\frac{3}{4} \beta^{2}=0 \\
\frac{\ddot{b}}{\bar{b}}+\frac{\dot{b}^{2}}{b^{2}}-\frac{1}{b^{2}}=\lambda \\
\frac{\ddot{b}}{\bar{b}}+\frac{\dot{b}^{2}}{b^{2}}-\frac{1}{b^{2}}=0 \\
\frac{\ddot{b}}{b}+\frac{\dot{b}^{2}}{b^{2}}-\frac{1}{b^{2}}=\rho
\end{gathered}
$$

Solving equation (45) we get,

$$
b=\left(t^{2}+2 k_{5} t+2 k_{6}\right)^{\frac{1}{2}}
$$

where, $k_{5}$ and $k_{6}$ are constants of integrations.

And equation (15) and (47) yields

$$
a=\left(t^{2}+2 k_{5} t+2 k_{6}\right)^{\frac{1}{2}}
$$


Equations (44), (45) and (46) gives $\rho=\lambda=0$, which satisfies the condition $\rho+\lambda=0$ and so the Energy density is given by

$$
\rho=0
$$

The tension density is given by

$$
\lambda=0
$$

And the rest Energy density is given by

$$
\rho_{p}=0
$$

This leads five dimensional vacuum model in Einstein's theory.

Thus the geometry of this model is described by the metric

$$
d s^{2}=-d t^{2}+\left(t^{2}+2 k_{5} t+2 k_{6}\right)\left(d x^{2}+e^{-2 x} d y^{2}\right)+k_{4}^{2} d z^{2}+k_{4}^{2 n} d m^{2}
$$

$$
\text { 3.3.CaseIII: } \frac{\dot{c}}{c}=0 \text { and } \frac{\ddot{c}}{\dot{c}}+n \frac{\dot{c}}{c}+2 \frac{\dot{b}}{b}=0 \text {. }
$$

This one case is not valid as the second equation becomes indeterminate.

Taking $k=-1, k_{1}=k_{2}=k_{3}=2, n=-0.5$ the variation of some parameters of the model (31) are shown below-

\section{Physical Interpretations of the solutions}

In case I, we have obtained a five dimensional Bianchi type-III string cosmological model universe in Lyra geometry given by the equation (31). The physical and geometrical behavior of model (31) are discussed as

(i) At initial epoch $t=0$, the metric (31) becomes flat. And as time increases and $-1<n<0$ the model expands along $\mathrm{z}$ axis but contract along $\mathrm{x}$ and $\mathrm{y}$ axes. The extra dimension contracts and becomes unobservable at $t \rightarrow \infty$.

(ii) Our model satisfies the energy density conditions $\rho \geq 0$ and $\rho_{p} \geq 0$. From the Fig.1, it is observed that the evolution of Energy density $\rho$ is infinite at time $t=0$ and it decreases as the time $t$ increases and become constant after some finite time.

(iii) From Fig.1 it is also observed that $\rho>0, \lambda<0$ and $\rho_{p}>0$, which shows that at early era strings exist with negative $\lambda$ but particles exist with positive $\rho_{p}$.

(iv) From the expansion scalar for the model (31), at initial epoch $t=0$, the expansion scalar $\theta$ is finite and as the time progresses gradually it decreases and finally $\theta$ becomes 0 when $t$ is infinite(as shown in the Fig.2). Hence the model shows that the universe is expanding with the progresses of time however the charge of expansion slower with respect to time and the expansion will stop at $t \rightarrow \infty$ and so the expansion is finite.

(v) Our model represents a shearing universe as shown in Fig.2, which shows a possible big crunch at some initial epoch $t=0$. Since $\frac{\sigma^{2}}{\theta^{2}} \neq 0$ as $t \rightarrow \infty$ and hence our model universe obtained here is anisotropic one. Though the anisotropy is included, it does not make any contradiction with the present day observational findings that the universe is isotropic one. This is due to the reason that during the process of evolution of our universe the initial anisotropy disappears after some epoch and approaches to the isotropy in late time universe.

(vi) The spatial volume $\mathrm{V}$ in the model (31) is finite at $t=0$, and it increases as $\mathrm{t}$ increases but in dumped way as shown in Fig.4.

(vii) The deceleration parameter $\mathrm{q}$ in (39) is decelerating at $t=0$ and it decreases as time increases and becomes negative after some finite time as shown in Fig.3, which indicates inflation in the model after an epoch of deceleration.

(viii) Equation (38) shows that when $t \rightarrow 0$ then $\mathrm{H}$ is constant but it decreases as $\mathrm{t}$ 


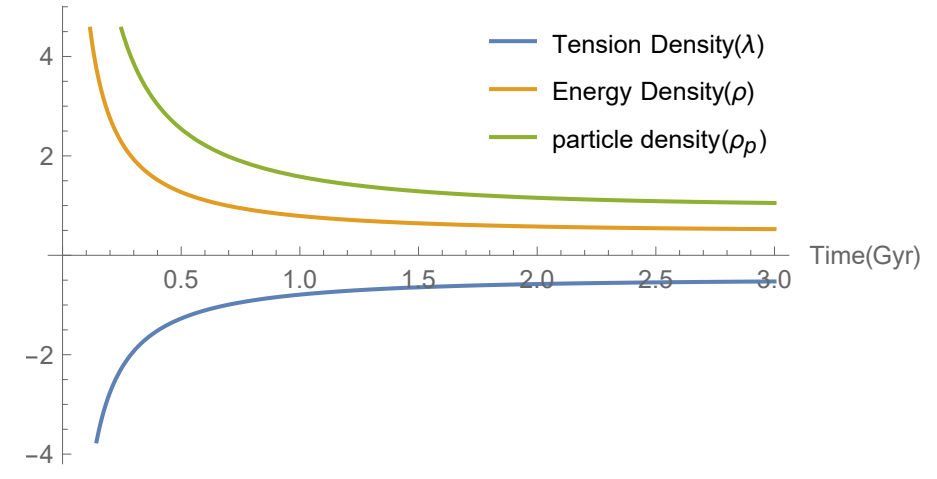

Figure 1. Variation of $\lambda, \rho, \rho_{p}$ vs. $t$.

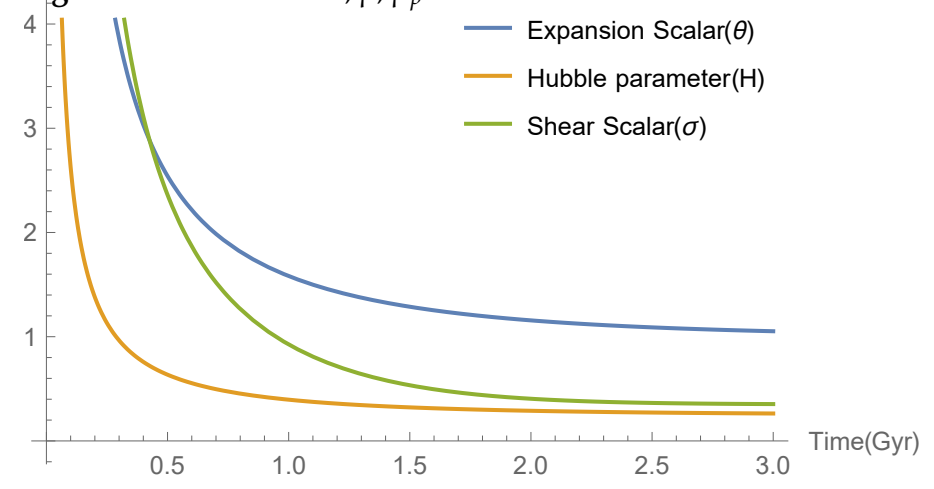

Figure 2. Variation of $\theta, \mathrm{H}, \sigma$ vs. $t$.

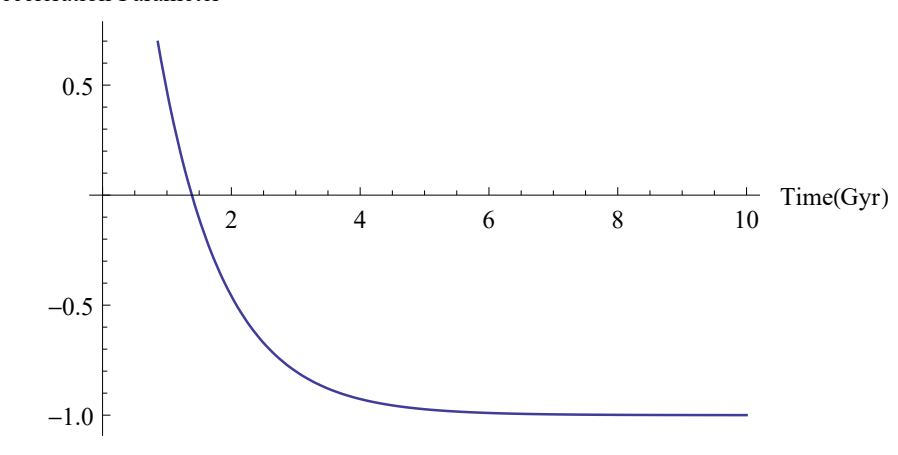

Figure 3. Variation of q vs. $t$.

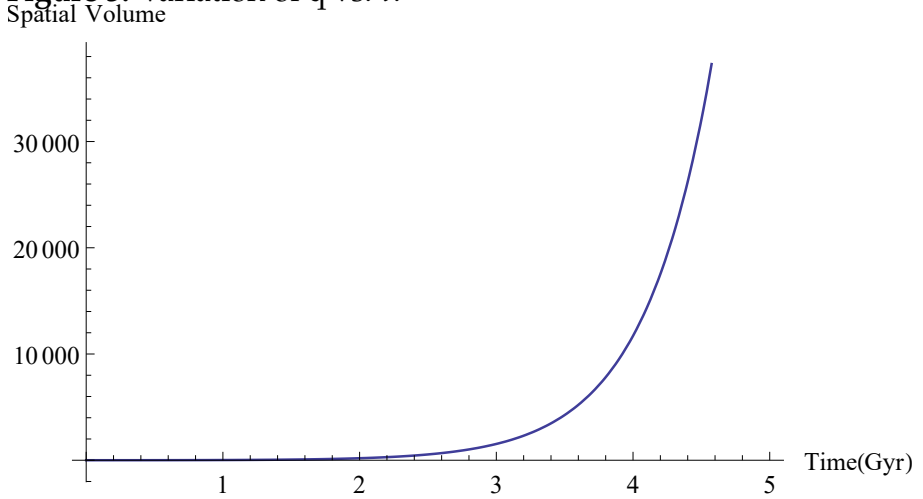

Figure 4. Variation of $\mathrm{V}$ vs. $t$. 
increases and finally when $t \rightarrow \infty$, then it vanishes as shown by Fig.2.

(ix) Also, from the Fig.1, it is seen that the particle density $\rho_{p}$ has a large value at the time of Big bang when $t=0$. As the evolution of time it decreases and reaches to a constant finite value at $t \rightarrow \infty$, which correspond to total constant finite number of particles in the late universe.

In case II, since $\rho=\lambda=0$, the strings don't survive and this leads five dimensional vacuum model in Einstein's theory of relativity. So, the model (52) represents a five dimensional Bianchi type -III vacuum cosmological model in general relativity.

\section{Conclusion}

Here, we have constructed a five dimensional Bianchi type-III String cosmological model in the framework of Lyra geometry given by (31). The physical and geometrical parameters which are very important in the discussion of cosmological models have been obtained and discussed. The model universe obtained here is expanding, anisotropic, shearing and accelerating one. We observed that our model is an inflationary model universe in which which is decelerating at initial epoch and accelerates after some finite time, indicating inflation in the model after an epoch of deceleration which is in accordance with the present day observational scenario of the accelerated expansion of our universe as type Ia supernovae [Riess et al.[46] and Perlmutter et al.[47]]. Our proposed model has a cosmological significance since it can explain the early stage of the universe at the early epoch. The model universe obtained here is anisotropic one in the early stage but it does not contradicts the recent observational findings that there is discrepancy in the measurement of intensities of microwaves coming from different directions of the sky, which urge us to study the universe with anisotropy Bianchi type-III metric in such a way to describe our universe in more realistic situation. Also, several cosmic microwave background $(\mathrm{CMB})$ anomalies such as inconsistency of the temperature anisotropies in the CMB with exact homogeneous and isotropic FRW model measured by COBE(Cosmic Background Explorer)/WMAP(Wilkinson Microwave Anisotropy Probe) satellites, foregrounds and exotic topologies are in evidence that we are living in a globally anisotropic universe. During the inflation, Shear scalar decreases and in due course it turns into an isotropic phase with very small value. As expected, in the present models the energy density and particle density remain positive. So, it satisfies the energy conditions, which shows that our models are physically realistic as the present day's observational data. Thus, we belief that the solutions and its interpretations presented in this paper may put some ideas on the understanding of the evolution of the early universe in further research.

\section{Acknowledgement}

K. Priyokumar Singh acknowledges CSIR, New Delhi, India for giving financial support to carry out this work successfully under the Research project Order No. F. 25(0279)/18/EMR-II of dated 4th April, 2018 .

\section{References}

1. J. Stachel,Thickening the string. I. The string perfect dust, Phys. Rev. D 21 (1980) 2171-2181.

2. P. S. Letelier,String Cosmologies, Phys. Rev. D 28 (1983) 2414-2419.

3. T. W. B. Kibble,Topology of cosmic domains and strings, J. Phys. A. Math. Gen. 9 (1976) 1387-1398.

4. T. W. B. Kibble,Some implications of a cosmological phase transition, Phys. Rept. 67 (1980) 183-199.

5. Y. B. Z. et al.,Cosmological consequences of spontaneous violation of discrete symmetry, Zh. Eksp. Teor. Fiz. 67 (1974) 3-11. 
6. Y. B. Z. et al., Cosmological fluctuations produced near a singularity, Mon. Not. R. Astron.Soc. 192 (1980) 663-667.

7. A. Vilenkin, Cosmic strings, Phys. Rev. D 24 (1981) 2082-2089.

8. A. Vilenkin,Gravitational field of vacuum domain walls and strings, Rev. D 23 (1981) 852-857.

9. G. Lyra, Über eine Modifikation der Riemannschen Geometrie, Mathematische Zeitschrift 54 (1951) 52-64.

10. F. Rahaman, J. Bera, Higher dimensional cosmological model in Lyra geometry, Int. J. Mod. Phys. 10 (2001) 729-733.

11. F. Rahaman, S. Chakraborty, J. Bera, Inhomogeneous cosmological model in Lyra geometry, Int J. Mod. Phys. 11 (2002) 1501-1504.

12. G. P. Singh, R. V. Deshpande, T. Singh,Higher-dimensional cosmological model with variable gravitational constant and bulk viscosity in Lyra geometry, Pramana J. Phys. 63 (2004) 937-945.

13. G. Mohanty, K. L. Mahanta, R. R. Sahoo, Non-existence of five dimensional perfect fluid cosmological model in Lyra manifold, Astrophys. space Sci. 306 (2006) 269-272.

14. G. Mohanty, K. L. Mahanta, B. K. Bishi,Five dimensional cosmological models in Lyra geometry with time dependent displacement field, Astrophys. space Sci. 310 (2007) 273-276

15. G. Mohanty, K. L. Mahanta, Five-dimensional axially symmetric string cosmological model in Lyra manifold, Astrophys. Space Sci. 312 (2007) 301-304.

16. P. Singh, M. Mollah,Higher Dimensional LRS Bianchi type - I Cosmological Model Universe Interacting with Perfect Fluid in Lyra Geometry, The African Rev. of phys. 11 (2016) 33-38.

17. F. Rahaman, S. Chakraborty, S. Das, M. Hossain, J. Bera, Higher-dimensional string theory in Lyra geometryPramana J. Phys. 60 (2003) 453-459.

18. G. Mahanty, G. C. Samanta,Five-dimensional string cosmological models with massive scalar field, FIZIKA B 19 (2010) 43-52.

19. V. K. Y. et al.,,Bianchi type-III anisotropic universes with a cloud of strings in Lyra's geometry Fizika B 19 (2010) 29-42.

20. G. C. Samanta, S. K. Biswal, G. Mohanty, Some five dimensional Bianchi type-III String Cosmological models in General Relativity, Bulg. J. Phys. 38 (2011) 380-389.

21. K. S. A. et al., N-dimensional string cosmological model in Brans-Dicke theory of gravitation, Astrophys. Space Sci. 310 (2007) 231-235.

22. K. et al.,Bianchi Type-III String Cosmological Models in The Presence of Magnetic Field in General Relativity, Int. J. Sci. Eng. Res. 3 (2012) 1-7.

23. Sahoo, P.K.,Nath,A.,Sahu, S.K.: Bianchi type-III String Cosmological model with bulk viscous fluid in lyra geometry ,Ira J. Sci. Technol. Trans Sci.Vol.41,(2017) pp.243-248 .

24. D. R. K. Reddy, Plane Symmetric Cosmic Strings In Lyra Manifold., Astrophys. Space Sci. 300 (2005) 381-386.

25. D. R. K. Reddy, R. L. Naidu, Five dimensional string cosmological models in a scalar-tensor theory of gravitation, Astrophys. Space Sci. 307 (2007) 395-398.

26. G. S. Khadekar, S. D. Tade, String cosmological models in five dimensional bimetric theory of gravitation, Astrophys. Space Sci. 310 (2007) 47-51.

27. V. U. M. Rao, T. Vinutha, M. V. Santhi, Bianchi type-V cosmological model with perfect fluid using negative constant deceleration parameter in a scalar tensor theory based on Lyra Manifold, Astrophys. Space Sci. 314 (2008) 213-216.

28. A. K. Yadav, V. K. Yadav, L. Yadav, Bianchi type-V string cosmological models in general relativity, Astrophys. Space Sci. 76 (2011) 681-690.

29. G. K. G. et al, Anisotropic universe with magnetized dark energy Astrophys. Space Sci. 361 (2016) 1-10.

30. G. P. S. et al.,Bianchi type-I bulk viscous cosmology with Chaplygin gas in Lyra Geometry, Chin. J. Phys. 54 (2016) 895-905.

31. B. R. Tripathi, A. Tyagi, S. Parikh,Bianchi Type-I Inhomogeneous String Cosmological Model with Electromagnetic Field in General Relativity, Prespacetime J. 8 (2017) 474-483.

32. V. F. P. et al.,Bianchi type II cosmological model of the Universe's evolution, Int. J. Geom. Meth. Mod. Phys. 15 (2018) 11850016.

33. A. Pradhan, R. Jaiswal, Magnetized string cosmological models of accelerated expansion of the Universe in theory $f(R, T)$ of gravity, Int. J. Geom. Meth. Mod. Phys. 15 (2018) 51850028.

34. R. K. Tiwari, B. K. Shukla, S. Mishra, Bianchi Type III String Cosmological Model in f (R, T) Modified Gravity Theory, Prespacetime J. 10 (2019) 306-315. 
35. S. Ram, M. K. Verma, Spatially Homogeneous Bianchi Type I Mesonic Models in Two-Fluid Cosmology, Prespacetime J. 10 (2019) 293-300.

36. M. R. Mollah, P. K. Singh, P. S. Singh,On Bianchi Type III Cosmological Model with quadratic EoS in Lyra Geometry, Int. J. Geom. Methods Mod. Phys, Int.J.Geom. Meth. Mod. Phys. 15 (2019) 185-194.

37. D. K. Sen, A static cosmological model, Z.Phys. 149 (1957) 311-323.

38. K. S. Thorne,Primordial element formation, primordial magnetic fields, and the isotropy of the universe, Astrophys. Space Sci. 148 (1967) 51-68.

39. R. Kantowski, R. K. Sachs,Some spatially homogeneous anisotropic relativistic cosmological models, J.Math. Phys. 7 (1966) 443-446.

40. J. Kristian, R. K. Sachs, Observations in cosmology, Astrophys.J. 143 (1966) 379-399.

41. C. B. Collins, E. N. Glass, D. A. Wilkinson,Exact spatially homogeneous cosmologies, Gen. Rel. Gravit. 12 (1980) 805-823.

42. D. R. K. Reddy,A string cosmological model in a scalar - Tensor theory of gravitation, Astrophys Space Sci. 286 (2003) 359-363.

43. D. R. K. Reddy,A String cosmological model in Brans-Dicke theory of gravitation, Astrophys. Space Sci. 286 (2003) 365-371.

44. D. R. K. Reddy, M. V. S. Rao, A Xially Symmetric Cosmic Strings and Domain Walls in Lyra Geometry, Astrophys. space Sci. 302 (2006) 157-160.

45. D. R. K. Reddy, M. V. S. Rao,Axially Symmetric String Cosmological Model In Brans-Dicke Theory of Gravitation, Astrophys. Space Sci. 305 (2006) 183-186.

46. A. R. et al., OBSERVATIONAL EVIDENCE FROM SUPERNOVAE FOR AN ACCELERATING UNIVERSE AND A COSMOLOGICAL CONSTANT, Astron. J. 116 (1998) 1009-1038.

47. S. P. et al.,MEASUREMENTS OF $\Omega$ AND $\Lambda$ FROM 42 HIGH-REDSHIFT SUPERNOVAE, Astrophys. J. 517 (1999) 565-586. 
